\title{
tenons en fibres de quartz et RCR esthétiques
}

RÉSUMÉ Les tenons radiculaires en fibres de quartz, de par leurs nombreuses qualités sur le plan mécanique, biologique et esthétique, sont préférentiellement indiqués dans plusieurs situations cliniques. L'article se propose, à travers deux cas cliniques, de décrire deux modes d'utilisation de ce type de tenons. Le but est aussi de critiquer ces deux techniques en les comparant avec les autres techniques de reconstitutions corono-radiculaires esthétiques.

Amal IDRISSI JANATI

Médecin dentiste résidente en prothèse fixée,

Faculté de médecine dentaire de Rabat, Université Mohammed V,

Centre de consultation

et de traitement dentaires (CCTD)

de Rabat.

\section{Amal EL YAMANI}

Professeur agrégée en prothèse fixée, Faculté de médecine dentaire de Rabat, Université Mohammed V,

Centre de consultation

et de traitement dentaires (CCTD)

de Rabat.

\section{Jamal EL BERNOUSSI}

Professeur d'enseignement supérieur en prothèse fixée,

Faculté de médecine dentaire de Rabat, Université Mohammed V,

Centre de consultation

et de traitement dentaires (CCTD)

de Rabat.

\section{MOTS CLÉS}

tenon radiculaire

fibres de quartz

reconstitution corono-radiculaire 


\section{introduction}

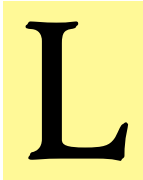

ongtemps, les reconstitutions corono-radiculaires (RCR) n'étaient pas concernées par le souci esthétique dans la mesure où elles ne constituent qu'une infrastructure à des couronnes ou bridges métalliques et/ou céramo- métalliques.

Désormais, les vingt dernières années ont vu de nombreux progrès dans le domaine des matériaux de restaura- tions esthétiques qui ont changé les modalités de traitement[1].

Avec l'utilisation des couronnes céramocéramiques translucides, type Empress ${ }^{\circledR}$, l'impact des moignons dentaires et prothétiques sur le résultat esthétique final est de plus en plus ressenti. Les céramiques alumineuses Inceram $^{\circledR}$ ou Procéra ${ }^{\circledR}$, beaucoup plus opaques imposent moins de contraintes esthétiques pour les restaurations sous jacentes[2].
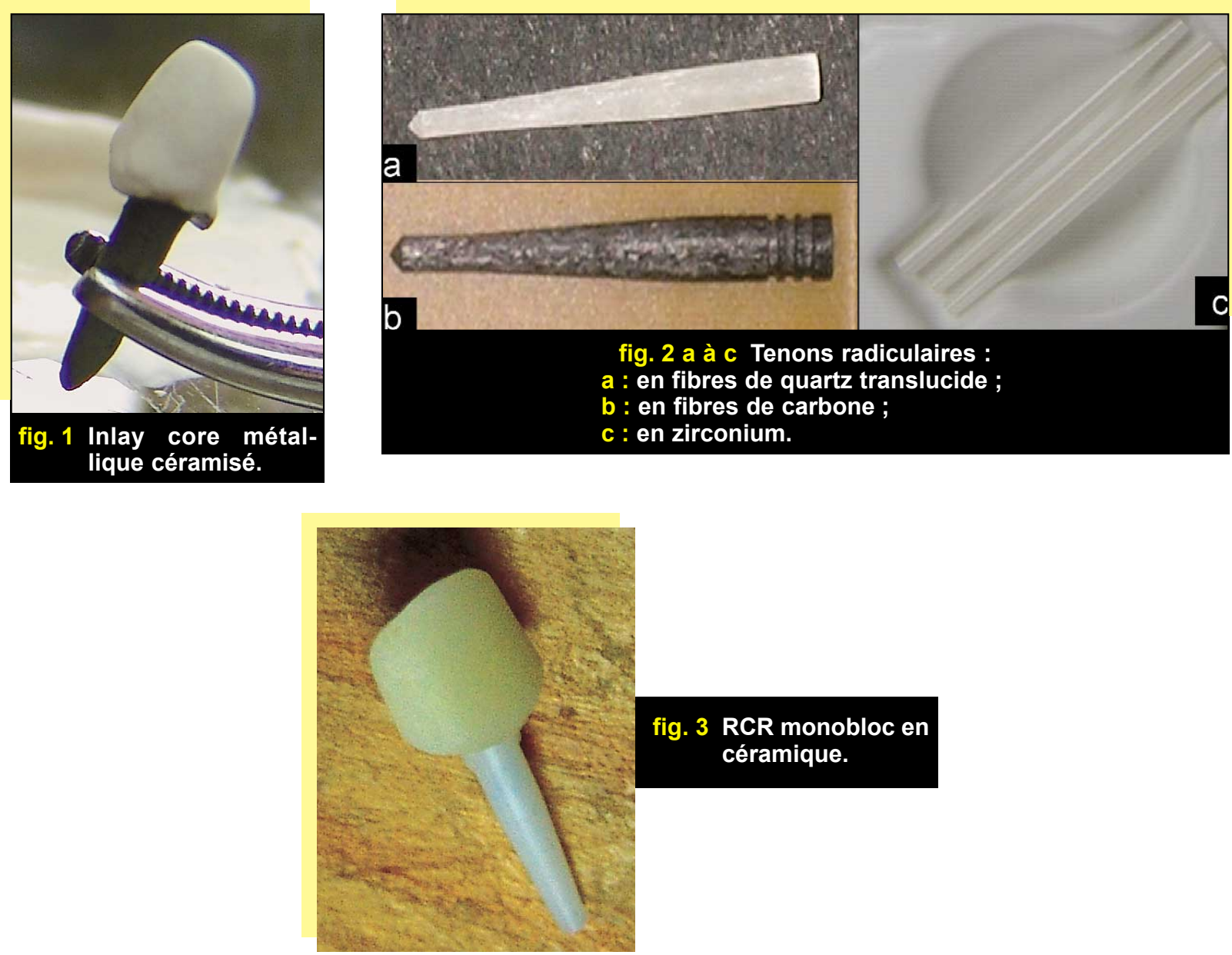
L'inlay-core métallique céramisé est le premier moyen proposé pour masquer l'infrastructure métallique. Il consiste à recouvrir la partie coronaire de l'inlaycore d'une couche de céramique opaque ou même de céramique dentine pour permettre les retouches cliniques (fig. 1). D'autres techniques proposent de supprimer entièrement le métal de la $\mathrm{RCR}$, et ce par l'utilisation des tenons en zirconium et des tenons fibrés, particulièrement les tenons en fibres de quartz ou de verre. Les tenons en fibres de carbone, de couleur marron foncé risquent de compromettre le rendu esthétique si le canal est large ou le parodonte est fin en créant un effet d'ombre au niveau cervical (fig. 2).

La technique dite entièrement céramique, elle, consiste à presser de la céramique Empress sur un tenon préfabriqué en oxyde de zirconium (ZrO2) pour obtenir une RCR monobloc en céramique (fig. 3 ).

Nous allons à travers cet article nous attarder sur deux techniques différentes de RCR esthétiques, utilisant les tenons en fibres de quartz.

Nous montrons leurs avantages et leurs limites ainsi que leurs indications, en les illustrant par deux cas cliniques.

\section{les tenons en fibres de quartz}

- composition et présentation

Les tenons en fibres de quartz sont fabriqués à partir de fibres longitudinales de quartz, parfaitement parallèles et liées entre elles par une matrice en résine époxy ou polyester. La teneur en fibres varie entre 60 et $65 \%$.
Il s'agit de tenons préfabriqués blancs, transparents ou opaques, qui se présentent sous différentes formes (cylindriques à doubles étages coniques, cylindriques, cylindro-coniques) (fig. 4) et différents diamètres (généralement trois ou quatre diamètres) (fig. 5).

Les tenons présentent des aspérités de

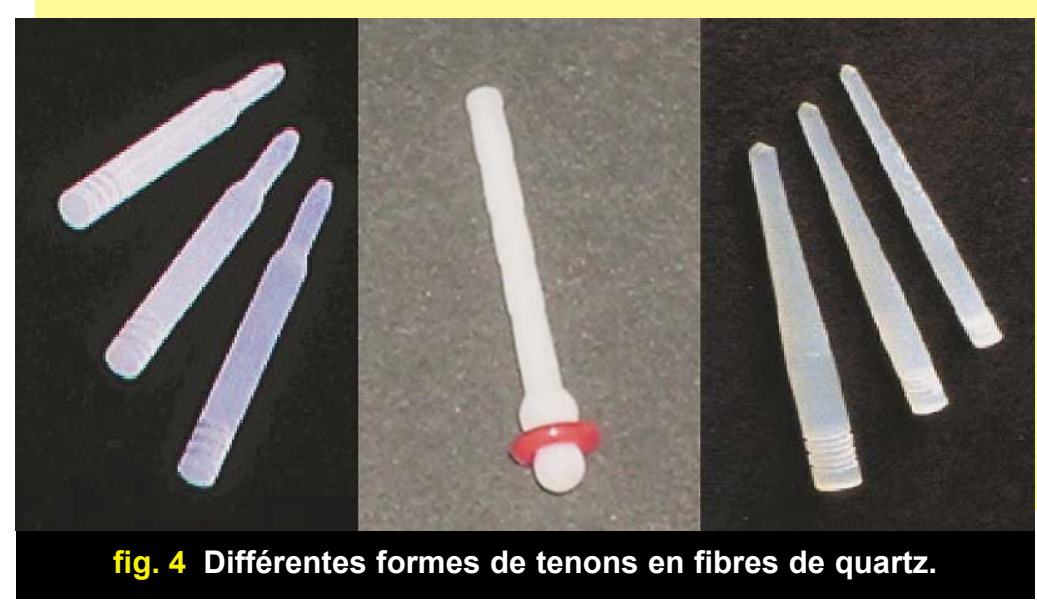




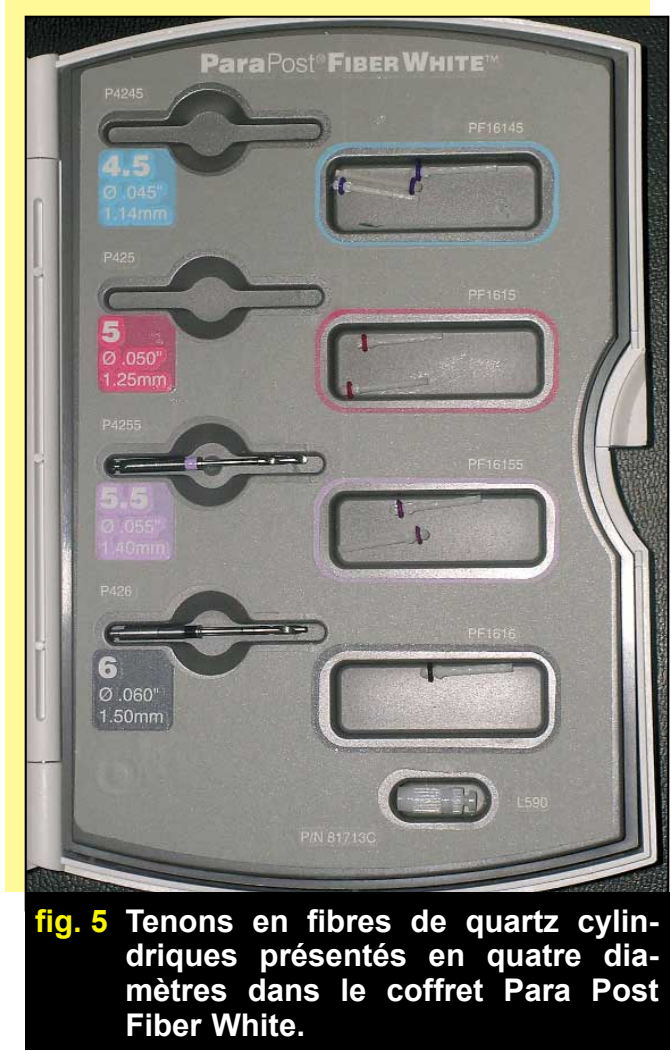

surface destinées à renforcer le collage. Ils sont généralement fournis dans des coffrets avec leurs forets adaptés qui permettent la mise en forme du logement canalaire. Certaines firmes proposent des trousses complètes : les tenons avec leurs alésoirs calibrés, le composite de collage et le composite de reconstitution (coffret D.T. Light-Post ${ }^{\circledR}$ de RTD (fig. 6).

\section{- avantages}

Les tenons en fibres de quartz allient un meilleur comportement mécanique, une esthétique très satisfaisante et une parfaite biocompatibilité.

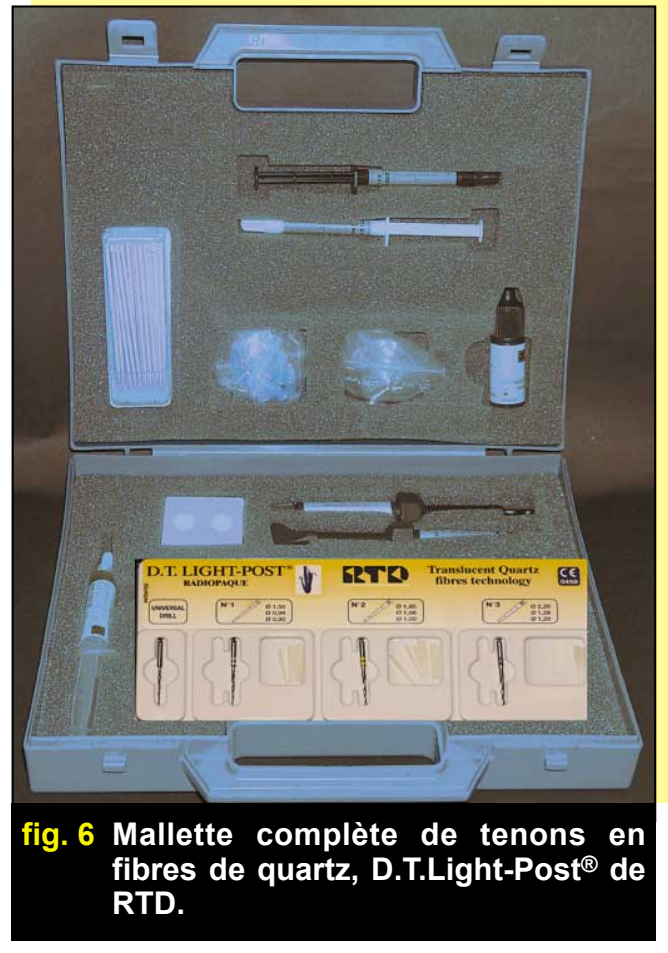

\section{SUR LE PLAN MÉCANIQUE}

Sachant que le tenon transmet intégralement les contraintes de la mastication à la dentine radiculaire, lorsque son module d'élasticité est élevé, il absorbe les déformations et transmet les contraintes brutalement sans les répartir. Celles-ci se concentrent alors au niveau de la dentine radiculaire dont la résistance est notablement inférieure à celle de la dentine coronaire. Dans le cas contraire, le tenon distribue les contraintes en les répartissant au sein de la totalité de la structure dentinaire, reproduisant ainsi un état très proche de l'état initial (dent pulpée), sans concentration excessive des contraintes. 
Ainsi, les tenons métalliques, notamment en or et en nickel chrome, dont le module d'élasticité est 5 à 10 fois supérieur à celui de la dentine (100 à 200 $\mathrm{GPa})$, sont souvent à l'origine de fissures puis de fractures radiculaires[3].

Les tenons en zirconium constituent un réel danger quant à leur utilisation si on considère leur module d'élasticité encore plus élevé que celui des tenons métalliques (200 GPa).

Les tenons en fibres de quartz, possédant un module d'élasticité très proche de celui de la dentine (de 18 à $50 \mathrm{GPa}$ ), semblent une alternative fiable aux tenons métalliques et ceux en zirconium. De par leur faible rigidité vis-àvis des structures dentaires, ils exposent moins au risque de fracture radiculaire que les autres tenons.

Notons aussi que leur résistance en flexion se situe dans un intervalle de 1400 à $1800 \mathrm{MPa}$, comparativement à $800 \mathrm{MPa}$ pour les pivots d'acier inoxydable et $1000 \mathrm{MPa}$ pour ceux en titanium.

\section{SUR LE PLAN BIOLOGIQUE}

Les tenons en fibres de quartz présentent un meilleur comportement biologique que les tenons métalliques.

Les risques de corrosion ou d'allergie à certains métaux nous poussent à nous orienter vers la suppression de toute infrastructure métallique dans nos réhabilitations prothétiques[4].

Rappelons que la corrosion des tenons métalliques a également été invoquée comme cause de fractures radiculaires[5].

Enfin, la présence d'un objet métallique, lors de certains examens médi- caux comme l'imagerie par résonance magnétique rend les résultats inexploitables.

\section{SUR LE PLAN ESTHÉTIQUE}

Dans le secteur antérieur, souvent exposé, la restitution d'une teinte et d'une anatomie correctes est parfois insuffisante.

Certaines situations sont considérées défavorables et exposent à un «risque esthétique». C'est le cas d'un parodonte fin, de tissus dentaires résiduels colorés ou d'une perte de substance dentaire importante qui laisse un logement de tenon anormalement large. Ces difficultés sont généralement rencontrées dans le cas de réfection d'anciennes reconstitutions.

Afin d'optimiser le résultat, ou à défaut de limiter l'aspect disgracieux, la RCR ne doit pas contribuer à assombrir la zone cervicale ni à modifier la teinte de la couronne. Un inlay-core métallique ou un tenon noir en fibres de carbone montre une tendance à griser la racine qui va accentuer l'effet d'ombre cervical.

Signalons aussi que des colorations de la gencive marginale peuvent être causées par la diffusion des débris de corrosion ou de débris métalliques lors de la retouche d'inlay-core[6].

Les tenons clairs en fibres de quartz contribuent efficacement, autant que les tenons en zircone, à l'éclaircissement de la région cervicale et à la diffusion de la lumière.

\section{SUR LE PLAN PRATIQUE}

La dépose, risquée pour les inlays-cores métalliques et quasi impossible pour les tenons en zirconium, est facile à envisager pour les tenons fibrés qui peuvent 
être fraisés avec un instrument rotatif adéquat, permettant ainsi toute éventuelle réintervention endodontique.

Rappelons aussi que la version translucide des tenons (Endo Light Post ${ }^{\circledR}$, RTD) offre en plus de la dimension esthétique requise, un gain de temps offert par la photopolymérisation à travers les tenons. L'utilisation des composites de scellement dual-cures peut-être envisagée.

\section{inconvénients}

Les tenons en fibres de quartz présentent une plus grande rigidité dans le sens axial par rapport aux tenons en fibres de carbone, ce qui les laisse préférer au niveau des dents antérieures où le problème devient secondaire, compte tenu de la direction des forces et de leur positionnement au sein du matériau composite[7].

\section{RCR avec tenon en fibres de quartz}

Il est actuellement admis que le tenon ne renforce pas la racine. Il constitue, au contraire, la principale étiologie des fractures radiculaires d'origine prothétique.

Son indication doit se limiter aux situations où plus de la moitié du tissu dentaire coronaire d'une dent dépulpée manque et où la dentine restante n'offre pas de possibilité de retenir correctement le matériau de reconstitution[1].

Parmi ces indications, le tenon en fibres de quartz est préférablement utilisé au niveau des dents antérieures vu sa plus grande rigidité dans le sens axial[7].

Deux modes d'utilisation sont possibles : l'un est directement réalisé au fauteuil et l'autre nécessite une étape de réalisation au laboratoire.

Les facteurs qui influencent le choix sont essentiellement le degré du délabrement coronaire et l'intensité des forces occlusales[8].

Ainsi, le choix entre les deux modes de reconstitution ne pourra se faire qu'après avoir défini, au niveau des parois axiales externes de la dent, les formes de contour définitives et le profil cervical choisi[9].

\section{- RCR foulée au tenon en fibres de quartz}

La technique consiste à sceller ou préférablement à coller un tenon en fibres de quartz dans son logement radiculaire. La partie du tenon, émergeant dans la cavité pulpaire, permet la rétention du composite de reconstitution qui vient obturer la cavité coronaire.

Les indications des RCR directes restent limitées aux pertes de substance moyennes en présence d'une occlusion favorable.

Les limites cervicales doivent être supragingivales et la quantité des pans dentinaires suffisante pour optimiser l'adhésion et la résistance aux contraintes du composite de reconstitution[7]. 


\section{cas clinique $\mathbf{n}^{\circ} \mathbf{1}$}

Une jeune patiente consulte pour la restauration de son incisive centrale, la 21 , fracturée et dyschromiée, suite à la nécrose. L'examen occlusal montre une occlusion en bout à bout avec l'absence de guidage antérieur (fig. 7). Le traitement canalaire est d'abord entrepris.

\section{- choix de la technique foulée}

Ce n'est qu'après préparation coronopériphérique en vue de recevoir une couronne céramo-céramique translucide en Empress $^{\circledR}$ que le délabrement coronaire est évalué.

Le nombre et la hauteur des parois coronaires restantes exigent une RCR, qui se doit d'être esthétique puisqu'elle sera recouverte d'une couronne translucide.

La situation supragingivale des pans dentinaires restants et la faible sollicitation occlusale de la dent ont plaidé en faveur d'une RCR foulée même si les parois restantes ne sont pas suffisamment hautes.

\section{- étapes de la RCR}

La préparation canalaire débute par un forage séquentiel alternant les forets Gates et Largo. Actuellement, on tend vers des diamètres compris entre 1 et $1,3 \mathrm{~mm}$. Cette épargne tissulaire ménage des épaisseurs radiculaires résistantes.

La longueur du forage doit être la plus élevée possible pour répartir uniformément les contraintes radiculaires et assurer une rétention maximale[10].

La mise en forme canalaire est ensuite obtenue grâce au foret calibreur adapté au diamètre du tenon choisi (fig. 8). Il permet un surdimensionnement $d u$ logement canalaire de quelques microns (30 microns) par rapport au diamètre
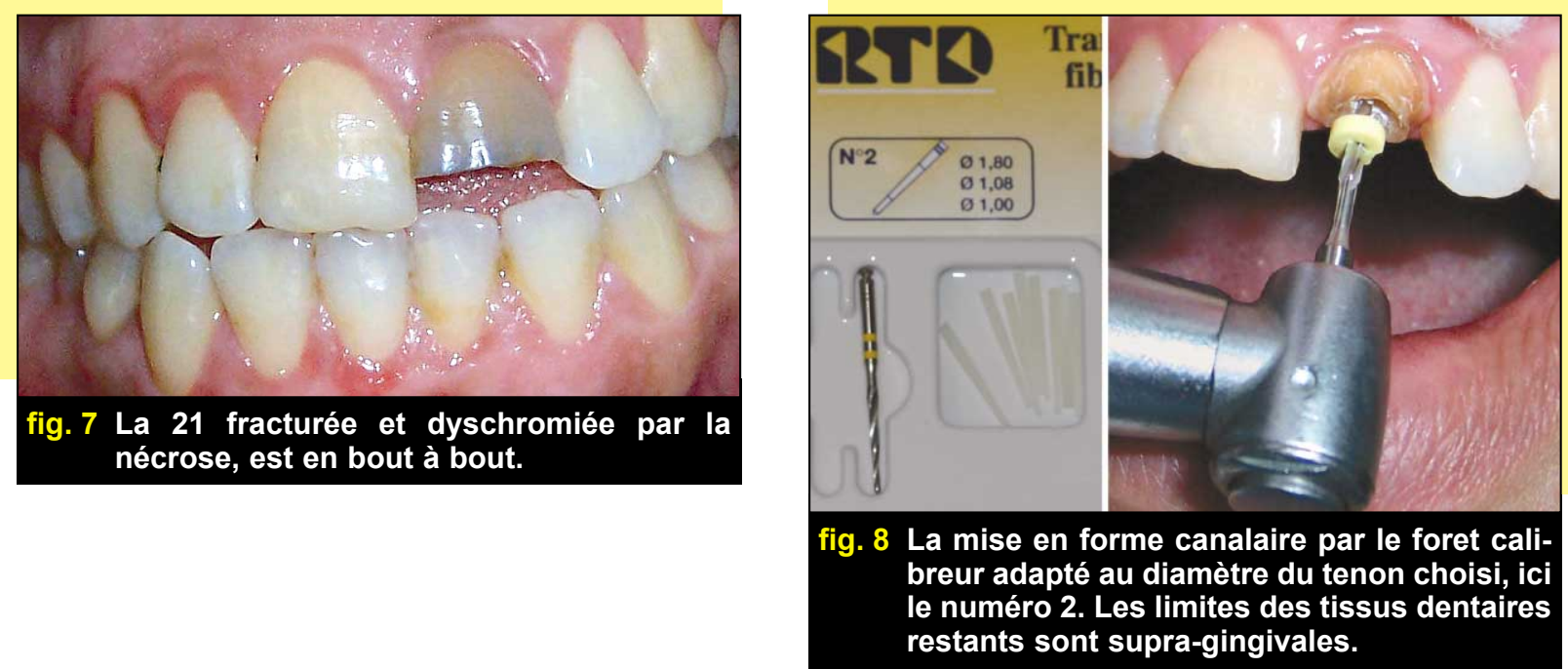

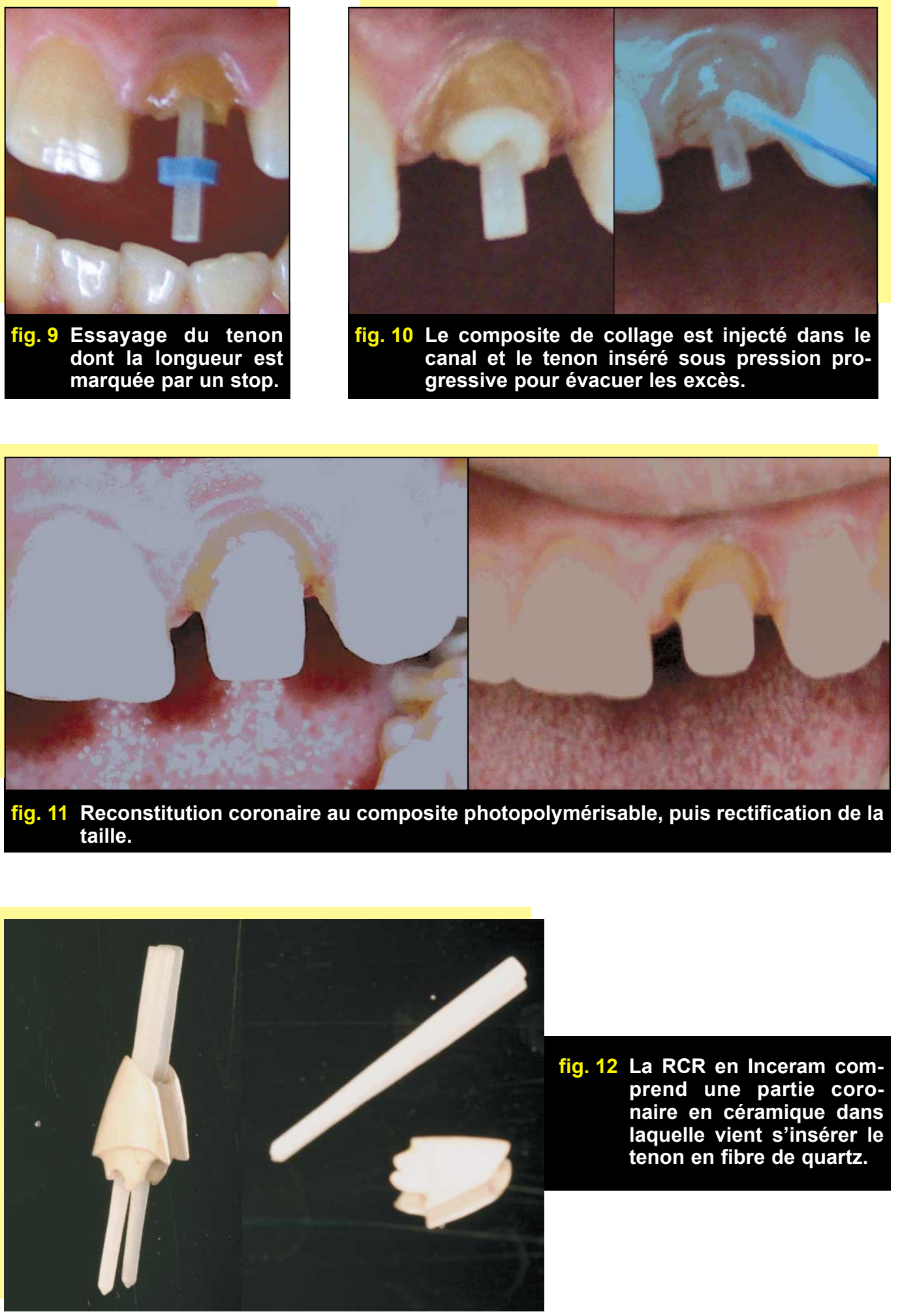

fig. 12 La RCR en Inceram comprend une partie coronaire en céramique dans laquelle vient s'insérer le tenon en fibre de quartz. 
du tenon. Cet espace est prévu pour le matériau d'assemblage.

Le tenon est d'abord essayé, et la longueur est marquée par un stop pour être sectionnée (fig. 9).

Les zones de contre-dépouilles doivent être conservées pour retenir le matériau de reconstitution coronaire[9].

Nous spécifions que nous avons utilisé la mallette des tenons en fibres de quartz Endo Light Post ${ }^{\circledR}$ de chez RTD : tenons translucides, alésoirs, système adhésif one step photopolymérisable, composite de collage et composite de reconstitution.

Le système adhésif utilisé préconise d'abord un prétraitement de la dentine intra-radiculaire et intra-coronaire à l'acide orthophosphorique à $37 \%$ laissé en contact des tissus durs pendant 10 secondes. Suivra un rinçage abondant pendant au moins une minute et un séchage léger.

L'adhésif, photopolymérisable, est apporté dans le canal à l'aide d'un pinceau en veillant à éliminer l'excès, avec cône en papier, avant la photopolymérisation. Il est aussi appliqué sur le tenon puis photopolymérisé.

Le composite de collage, bipolymérisant et fluide est injecté dans le canal grâce à un bourre pâte. Le tenon est ensuite inséré sous pression progressive pour évacuer les excès qui sont éliminés avant photopolymérisation (fig. 10).

La reconstitution coronaire est réalisée par un seul apport de composite autopolymérisable dans un moule odus, ou par apport de couches successives du composite de reconstitution photopolymérisable (fig. 11). Celui-ci peut être travaillé sitôt la polymérisation achevée. La préparation peut donc être immédiatement rectifiée[1]. L'empreinte secondaire est prise dans la même séance.

\section{- RCR en Inceram et tenon en fibres de quartz claveté}

La technique Inceram propose des reconstitutions en deux parties: une partie coronaire en céramique à travers laquelle vient s'insérer le tenon en fibre de quartz (fig. 12). La céramique doit pénétrer de quelques dixièmes de millimètres dans le logement canalaire afin de renforcer le tenon et de contribuer à l'esthétique cervicale[1].

La technique est indiquée même devant des délabrements coronaires très importants mais avec des limites cervicales juxta ou supra-gingivales, pour des impératifs de collage. La céramique résiste mieux aux fortes sollicitations occlusales que le composite en technique foulée.

\section{cas clinique $n^{\circ} 2$}

Une jeune patiente se présente pour la réfection de son ancienne prothèse, sur la 22. Sa motivation est surtout d'ordre esthétique. La prothèse s'avère aussi mal adaptée du point de vue parodontal et l'examen radiologique montre une obturation canalaire insuffisante, à l'origine d'une image péri-apicale. 
Lors de la première séance de consultation, nous avons réalisé une nouvelle couronne provisoire, afin de rétablir un meilleur état de la gencive marginale et de mettre en confiance la patiente en répondant à son souci esthétique (fig. 13 a et b). La reprise du traitement endodontique est réalisée lors de la séance suivante.

\section{- choix prothétique}

Le choix prothétique a été porté sur une couronne céramo-céramique translucide en Empress avec une reconstitution corono-radiculaire esthétique.

Le grand délabrement coronaire écartait la possibilité de toute reconstitution en composite foulé autour d'un ancrage radiculaire. La situation supra-gingivale des limites cervicales autorise le collage.

Une reconstitution coronaire indirecte en Inceram zirconia avec un tenon en fibres de quartz claveté est donc envisagée.
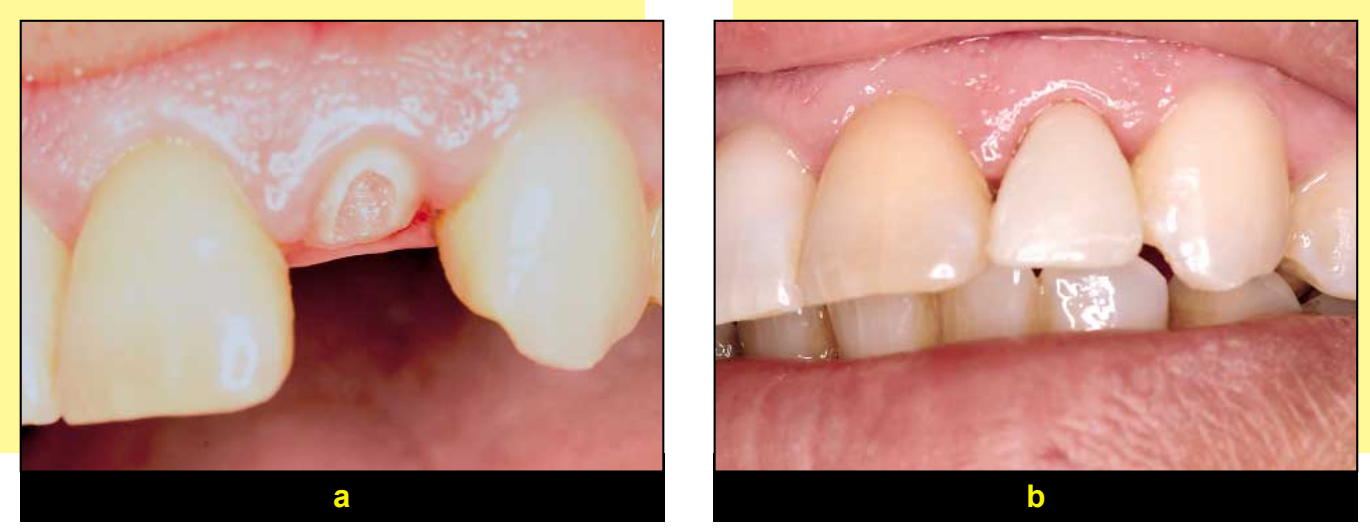

fig. 13 a et $b$

a : dépose de l'ancienne prothèse sur la 22 et rectification de la taille.

b : réalisation d'une nouvelle provisoire bien adaptée. 

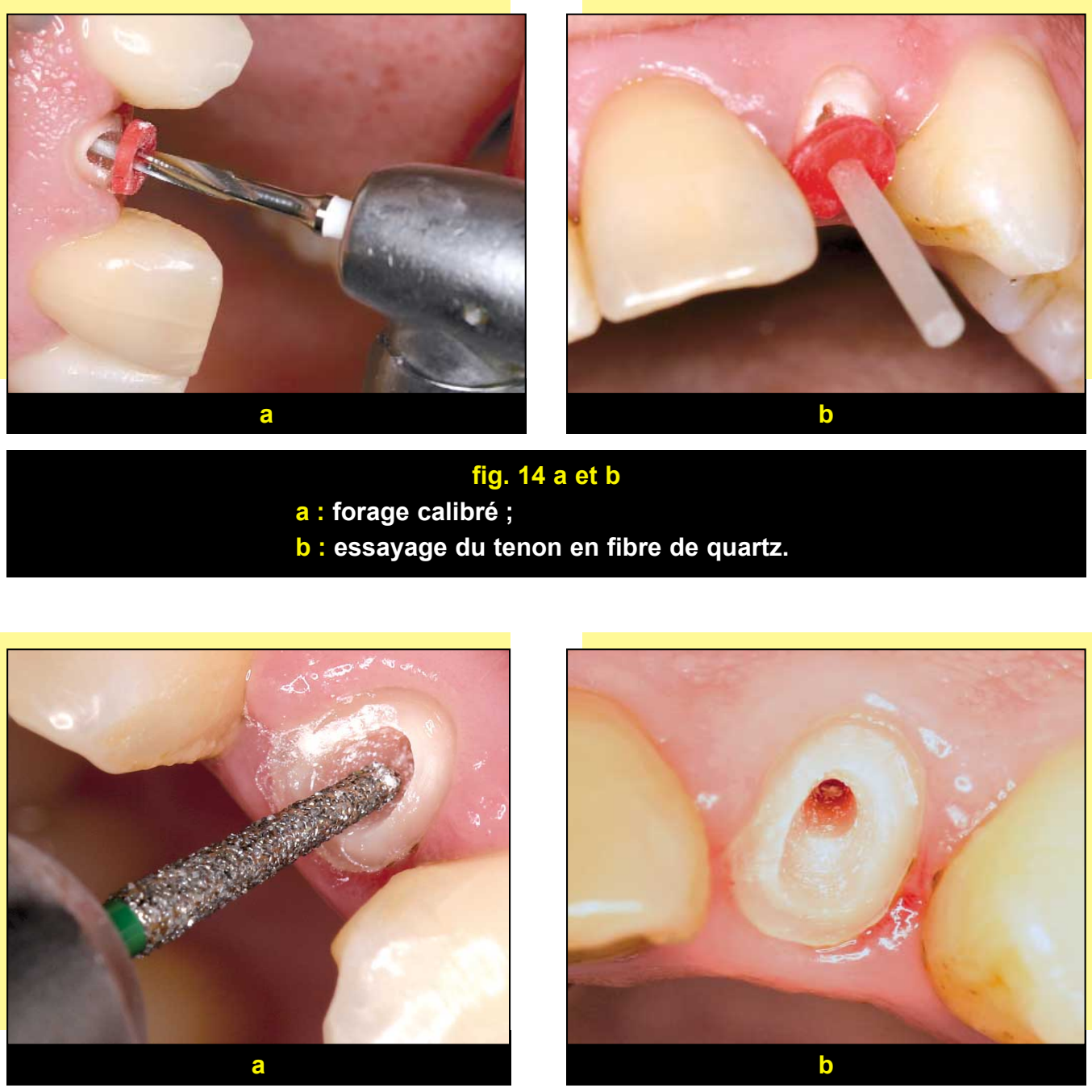

fig. 15 a et $b$

Une assise intra-canalaire est ménagée au niveau du tiers coronaire.

Une empreinte en double mélange est réalisée de façon classique. Elle est fournie au laboratoire de prothèse avec le tenon en fibre de quartz choisi. On prendra le soin de marquer à l'aide d'un stop le niveau de pénétration du tenon choisi pour que le prothésiste puisse vérifier la fiabilité de l'enregistrement clinique.
La prothèse provisoire, rebasée avec un tenon métallique, est scellée avec un ciment provisoire sans eugénol pour éviter d'interférer ultérieurement avec le composite de collage.

\section{DEUXIÈME ÉTAPE CLINIQUE}

L'inlay-core est d'abord essayé et validé (fig. 16 a), on procède aux séquences de 

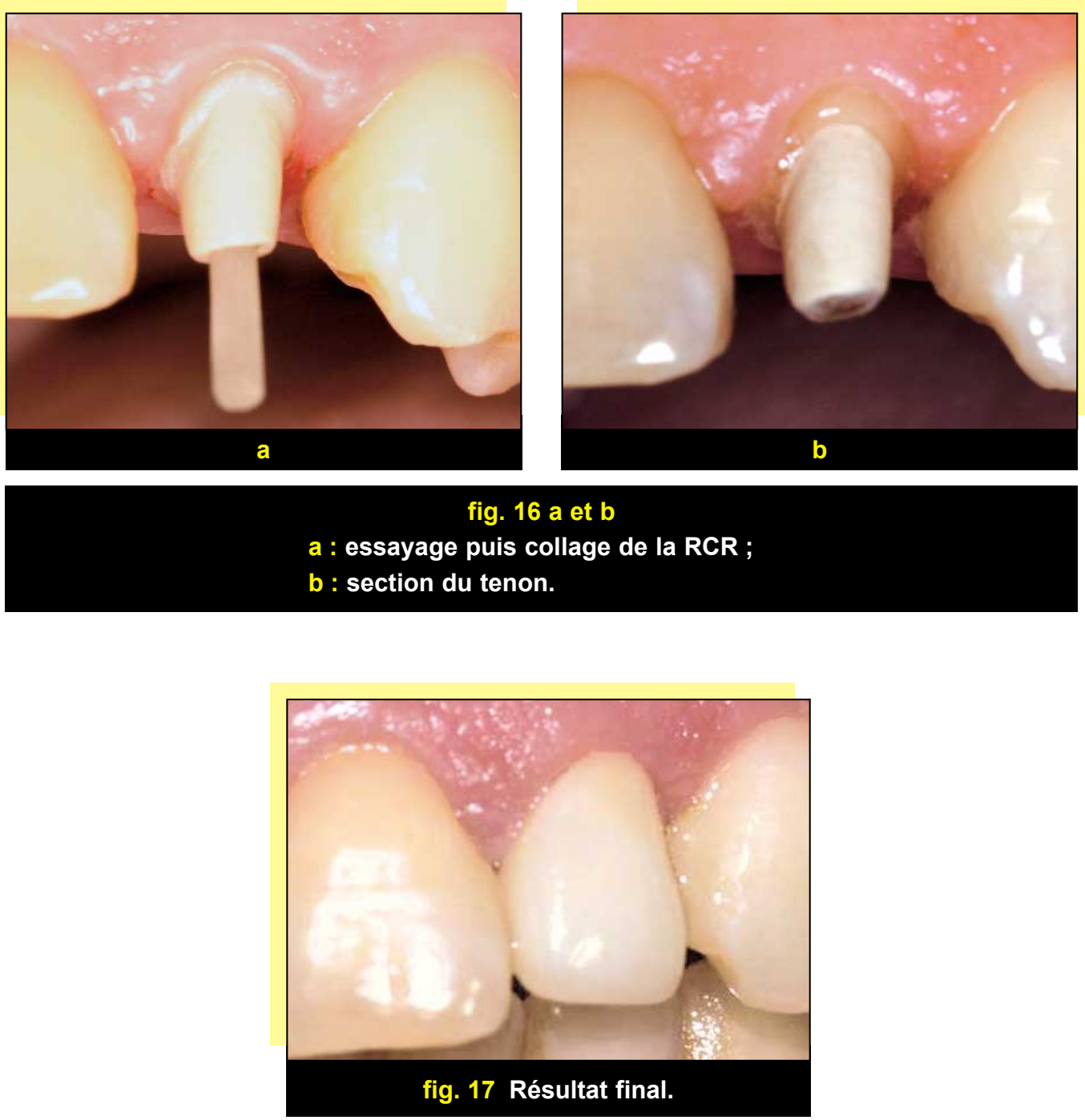

collage. Après mordançage canalaire, l'adhésif est appliqué puis photopolymérisé aussi bien dans le canal que sur le tenon et sur l'intrados de la pièce coronaire.

Le composite de collage est amené dans le canal avec un bourre pâte.

La partie coronaire, dont l'intrados est enduit de composite de collage, est mise en place en premier et le tenon est ensuite inséré sous pression progressive pour évacuer les excès.

L'ensemble est photopolymérisé en maintenant un appui sur l'inlay-core. Le tenon n'est sectionné qu'après collage pour faciliter la manipulation (fig. 16 b).

Le moignon peut être rectifié et l'empreinte secondaire peut être prise dans la même séance (fig. 17). 


\section{discussion}

- comparaison

entre les deux techniques

de RCR avec tenon

en fibre de quartz

La technique foulée, en préservant d'une part plus de dentine coronaire résiduelle et d'autre part, en utilisant un composite de reconstitution, un composite de collage et un tenon en fibres, offre un système cohérent, capable d'amortir et d'assimiler les forces occlusales en les répartissant au sein de la totalité de la structure dentaire[10].

Ne nécessitant qu'une seule étape clinique, la technique directe limite les erreurs cumulatives de plusieurs transferts entre la clinique et le laboratoire[3].

Désormais, la RCR foulée résiste moins bien aux fortes sollicitations occlusales, surtout les forces de flexion, que la RCR indirecte en Inceram, et ce grâce à l'utilisation de la céramique Inceram zirconia au niveau coronaire.

Il faut tout de même admettre que la technique indirecte est plus mutilante vis-à-vis des structures dentaires que la technique foulée et moins homogène. $\mathrm{Et}$ même si le protocole clinique est plus simple que celui de la technique foulée car il rejoint approximativement celui de l'inlay-core métallique, la réalisation $\mathrm{au}$ laboratoire de prothèses est très compliquée.

Dans les deux cas, l'utilisation du tenon en fibre de quartz est gage d'un meilleur comportement biomécanique de la reconstitution, d'une absence de corrosion, d'une possibilité de dépose et d'un résultat esthétique très satisfaisant[7]. De plus, le collage des tenons avec un composite spécifique crée un joint de colle qui augmente «l'effet tampon»[1].

Lorsqu'une RCR esthétique s'impose, le choix entre les deux techniques doit tenir compte du délabrement coronaire, de la situation des limites tissulaires et surtout de l'intensité des sollicitations occlusales. Devant une occlusion serrée, ou un délabrement coronaire très important ou des limites juxta-gingivales, la technique indirecte en Inceram avec tenon en fibres de quartz claveté sera de meilleure indication que la technique foulée.

\section{- comparaison}

avec les autres RCR esthétiques

Bien que les RCR en Inceram et les RCR en Empress se ressemblent quant à leur rendu esthétique et leur parfaite biocompatibilité, elles se voient très différentes quant à leurs comportements biomécaniques.

L'inlay-core en céramique monobloc, par l'utilisation du tenon en zirconium, est extrêmement rigide et peut transmettre les forces occlusales à la racine. Il est donc conseillé de le réserver aux dents qui ont des structures radiculaires suffisantes.

De plus, le démontage des inlays-cores collés est pratiquement impossible. La réintervention endodontique est de ce fait aussi quasi impossible. 
Vue sous cet angle, la technique en Inceram, utilisant des tenons en fibres de quartz moins rigides et facilement démontables est plus sûre.

Enfin, signalons que les RCR avec tenons en fibres de quartz présentent des limites quant à leurs utilisations. Une situation juxta ou sous-gingivale des limites cervicales ne permet pas d'entreprendre un procédé de collage fiable. Dans ce cas, l'inlay-core métallique céramisé est le seul moyen de RCR esthétique autorisé même si le résultat esthétique n'est pas toujours certain devant des situations de haut risque esthétique.

\section{conclusion}

Devant l'opacité des tenons en fibres de carbone et des tenons métalliques, et la grande rigidité des tenons en zirconium blancs, les tenons en fibres de quartz paraissent comme le meilleur compromis puisqu'ils assurent à la fois une bonne esthétique, un bon comportement biomécanique et une parfaite biocompatibilité. Cependant, leur utilisation doit être très rigoureuse et surtout bien justifiée.

\section{bibliographie}

1. Bolhuis Henricus PB, Pameijer Jan HN.

Reconstitutions préprothétiques pour couronnes et bridges. Réalités cliniques 2000 11(4):461-476.

2. Aboudharam $G$, Laurent $M$. Évolution et indications des reconstitutions corono-radiculaires indirectes.

Cahiers de Prothèse 2001; 116:61-71.

3. Pourreyron L, Abadie JM Étude au M.E.B. des composants et des interfaces d'un nouveau système de reconstruction corono-radiculaire. Cahiers de Prothèse 1993;84:32-46.

4. Calamand P, Pfeffer D. Reconstitutions prothétiques

"tout céramique" :

un concept novateur.

Clinic 1996;17(10):567-572.

5. Purton DG, Payne JA. Tenons radiculaires : fibre de carbone ou acier inoxydable ? Clinic 1996;17(5):275-279.

6. Gonthier $\mathrm{S}$

Desreumax-Gonthier M. Influence esthétique des reconstitutions corono-radiculaires. Réalités Cliniques 2003; 14(4):443-456.

7. Chafaie $A$ Reconstitution corono-radiculaire à l'aide de tenons à base de fibres de quartz et de résine composite.

Cahiers de Prothèse 2001; 116:83-87.
8. Pierrisnard $L$.

Conservation des dents délabrées et reconstitutions corono-radiculaires (consensus, évolution et perspectives). Chir Dent Fr 1999;952: 40-42.

9. Porzier J, Benner $L_{\text {, }}$ Radiguet J, Losfeld R. Reconstitutions préprothétiques immédiates des dents dépulpées. Cahiers de Prothèse 1988; 63:35-46.

10. Geoffrion J, Bartala M. Économie tissulaire et préparations des ancrages corono-radiculaires. Cahiers de Prothèse 2003; 124:63-70. 


\section{SUMMARY}

\section{Quartz radicular fibre post and aesthetic RCR}

A. IDRISSI JANATI, A. EL YAMANI, J. EL BERNOUSSI

Quartz radicular fibre post, of share their many qualities on the mechanical, biological and aesthetic level, are preferentially indicated in several clinical situations. The article proposes, through two clinical cases, to des-

cribe two modes of use of this type of posts. The goal is also to criticize these two techniques by comparing them with the other techniques of aesthetic corono-radicular reconstitutions.

keywords: radicular tenon, quartz fibres, corono-radicular reconstitution. 